\title{
Load optimization factors for analyzing the efficiency of wireless power transfer systems using two-port network parameters
}

\author{
Yoshiaki Narusue $^{1, \text { a)}}$, Yoshihiro Kawahara ${ }^{1}$, and Hiroyuki Morikawa ${ }^{1}$
}

\begin{abstract}
This paper models and analyzes the power efficiency of wireless power transfer systems with arbitrary load impedances using passive twoport network parameters. In this formulation, two novel indices-the load resistance optimization factor and the load reactance optimization factorare defined and introduced to indicate the deviation of the load resistance and load reactance from their optimal values. It is shown that the power efficiency of these systems can be formulated using only their $k-Q$ product and the defined load optimization factors. Additionally, we show that the ratio of efficiency to maximum available efficiency can be characterized with one single complex factor. The proposed approach helps the quantitive interpretation of the efficiency deterioration by the difference between the load impedance and its optimal value.

Keywords: efficiency formulation, passive two-port network, wireless power transfer

Classification: Microwave and millimeter-wave devices, circuits, and modules
\end{abstract}

\section{Introduction}

Electromagnetic wireless power transfer is becoming a key technology for powering electrical devices in the IoT (Internet of Things) era $[1,2,3,4,5,6]$, in which the disappearance of power supply cables will drastically change the ways of using electrical equipment.

Load impedance is a key parameter in the design of highefficiency wireless transmission systems, given the strong influence of this parameter on the overall system efficiency. The mainstream approach for the design and analysis of load impedance has traditionally been based on equivalent circuit analysis $[7,8,9,10,11,12,13]$. In recent years, on the other hand, approaches to determine the optimal load impedance and maximum available efficiency using passive two-port network parameters have been studied $[14,15,16,17,18$, 19].

The advantage of formulations based on passive two-port network parameters is that they can be applied regardless of circuit topology [15]. When using equivalent circuit analysis, it is necessary to first accurately identify the equivalent circuit of the target system and then formulate the solution for the equivalent circuit obtained in that particular case. In contrast, when passive two-port network parameters are used, the optimal load impedance and maximum available

${ }^{1}$ Graduate School of Engineering, University of Tokyo, Tokyo 1138656, Japan

a) narusue@mlab.t.u-tokyo.ac.jp

DOI: $10.1587 /$ elex.17.20200093

Received March 10, 2020

Accepted March 31, 2020

Publicized April 10, 2020

Copyedited May 25, 2020 efficiency can be derived from two-port network parameters such as, e.g., the $\boldsymbol{Z}$ and $\boldsymbol{S}$ parameters, regardless of circuit configuration and whether or not the coupling between the transmitter and receiver is microwave-based, inductive, or capacitive.

In practice, however, it is difficult to reach the maximum available efficiency determined from passive two-port network parameters, because these parameters change from moment to moment, depending on the relative positions of the transmitter and receiver, and it is therefore not easy to adjust the load impedance accordingly, so as to maintain an optimal impedance.

The difficulty in maintaining the optimal impedance is further increased by the fact that the receiver does not necessarily have mechanisms to adjust the values of load resistance and load reactance, and because the adjustment resolution of such mechanisms-even when they do exist-has limits. It is known that the load resistance can be adjusted by a DC/DC converter connected to a subsequent rectifier circuit stage [20,21], and methods to follow the optimal value have been actively studied [22, 23, 24, 25]. However, even with a DC/DC converter, the load resistance cannot be completely controlled to the optimal resistance value. This may lead to mismatches between the actual load resistance and its optimal value. Reactance adjustment mechanisms, on the other hand, are still in the research stage. Reactance adjustment by switching lumped reactors has been proposed [26, 27, 28, 29]. However, given that the adjustment resolution is correlated with the number of elements used in the mechanism, the resolution of the reactance adjustment function must be sacrificed to achieve compact circuit packaging. Therefore, even if a reactance adjusting mechanism exists, it is still necessary to allow for the existence of a difference between the load reactance and its optimal value.

In order to analyze wireless power transfer systems in detail, we therefore need not only to determine the optimal impedance and maximum available efficiency, but also to know the mechanism by which load impedance deviations influence efficiency. Specifically, it is necessary to clarify how much can the efficiency be deteriorated by the difference between the load impedance and its optimal value, and which parameters determine the degree of deterioration.

In this paper, the power efficiency of wireless power transfer systems with arbitrary load impedances is formulated with passive two-port network parameters. In this formulation, we define and introduce two novel indices, the load optimization factors, which determine how the deviations of 


$$
\begin{aligned}
\eta & =\frac{\left(r_{\mathrm{m}}^{2}+x_{\mathrm{m}}^{2}\right) r}{r_{11} r^{2}+\left[2 r_{11} r_{22}-\left(r_{\mathrm{m}}^{2}-x_{\mathrm{m}}^{2}\right)\right] r+\left[r_{11}\left(r_{22}^{2}+\left(x+x_{22}\right)^{2}\right)-r_{22}\left(r_{\mathrm{m}}^{2}-x_{\mathrm{m}}^{2}\right)-2 r_{\mathrm{m}} x_{\mathrm{m}}\left(x+x_{22}\right)\right]} \\
& =\frac{\left(r_{\mathrm{m}}^{2}+x_{\mathrm{m}}^{2}\right)}{r_{11} r+\left[2\left(r_{11} r_{22}-r_{\mathrm{m}}^{2}\right)+\left(r_{\mathrm{m}}^{2}+x_{\mathrm{m}}^{2}\right)\right]+\frac{\left(r_{11} r_{22}-r_{\mathrm{m}}^{2}\right)\left(r_{11} r_{22}+x_{\mathrm{m}}^{2}\right)+\left[r_{11}\left(x+x_{22}\right)-r_{\mathrm{m}} x_{\mathrm{m}}\right]^{2}}{r_{11} r}}
\end{aligned}
$$

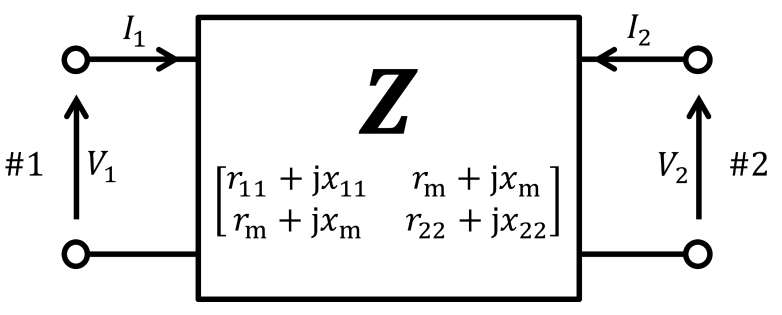

Fig. 1 Two-port network model, variables, and parameters

load resistance and load reactance from their optimal values affect power efficiency. These indices make it possible to easily analyze the efficiency degradation resulting from deviations in the load impedance.

\section{Efficiency definition}

This section analyses the power efficiency of wireless power transfer systems using passive two-port network parameters, and revisits the concepts of optimal impedance and maximum available efficiency.

A two-port network model and the associated variable definitions are shown in Fig. 1. In this figure, Port 1 is the power transmission side, Port 2 is the power reception side, and all voltages $\left(V_{1}\right.$ and $\left.V_{2}\right)$ and currents $\left(I_{1}\right.$ and $\left.I_{2}\right)$ are presented in phasor notation. In this paper, we will use the impedance parameters ( $\boldsymbol{Z}$ parameters) to analyze the twoport network. No generality is lost in doing so, because the passive two-port network parameters are easily interchangeable. For example, the $\boldsymbol{Z}$ parameters can be easily obtained from the scattering parameters ( $S$ parameters) that can be measured with a vector network analyzer, using the transformation

$$
\boldsymbol{Z}=z_{0}(\boldsymbol{I}+\boldsymbol{S})(\boldsymbol{I}-\boldsymbol{S})^{-1},
$$

where $z_{0}$ is the reference impedance of the $\boldsymbol{S}$ parameters, and $\boldsymbol{I}$ is the identity matrix [30].

In this paper, the efficiency $\eta$ is defined to be the power efficiency. Given that the input power (from the power transmission side) is $\operatorname{Re}\left(V_{1} I_{1}^{*}\right)$, and the power transmitted to the load is $\operatorname{Re}\left(\mathrm{V}_{2}\left(-\mathrm{I}_{2}^{*}\right)\right)$, the power efficiency is calculated as

$$
\eta=-\frac{\operatorname{Re}\left(V_{2} I_{2}^{*}\right)}{\operatorname{Re}\left(V_{1} I_{1}^{*}\right)},
$$

where $(\cdot)^{*}$ denotes the complex conjugate of $(\cdot)$.

By substituting the load impedance $z=r+\mathrm{j} x$ ( $r$ and $x$ are real variables) and the $\boldsymbol{Z}$ parameter variables, the power efficiency $\eta$ can be rewritten using passive two-port parameters as in Eq. (3). All the analysis and efficiency formulations in this paper will be based on Eq. (3).

Let us now revisit the concepts of optimal impedance and maximum available efficiency using passive two-port network parameters. The load impedance that maximizes the power efficiency, denoted by $z_{\mathrm{opt}}=r_{\mathrm{opt}}+\mathrm{jx} \mathrm{x}_{\mathrm{opt}}\left(r_{\mathrm{opt}}\right.$ and $x_{\text {opt }}$ are real variables), can be formulated as follows $[14,16]$ :

$$
\begin{aligned}
& r_{\mathrm{opt}}=\frac{|\boldsymbol{R}|}{r_{11}} \sqrt{1+(k Q)^{2}} \\
& x_{\mathrm{opt}}=\frac{r_{\mathrm{m}} x_{\mathrm{m}}-r_{11} x_{22}}{r_{11}},
\end{aligned}
$$

where $\boldsymbol{R}$ is the real part of $\boldsymbol{Z}$ and $(k Q)^{2}$ is the squared value of the $k-Q$ product. These quantities can be written in terms of the $\boldsymbol{Z}$ parameters, as follows [16]:

$$
\begin{aligned}
|\boldsymbol{R}| & =r_{11} r_{22}-r_{\mathrm{m}}^{2} \\
(k Q)^{2} & =\frac{r_{\mathrm{m}}^{2}+x_{\mathrm{m}}^{2}}{|\boldsymbol{R}|}
\end{aligned}
$$

The maximum available efficiency $\eta_{\mathrm{opt}}$ is obtained by substituting Eqs. (4) and (5) into Eq. (3), which results in

$$
\eta_{\mathrm{opt}}=\frac{(k Q)^{2}}{\left(1+\sqrt{1+(k Q)^{2}}\right)^{2}} \text {. }
$$

\section{Efficiency in the presence of load impedance devia- tions}

In this section, we first obtain the power efficiency $\eta_{\Delta x}$ in the case of an adjustable load resistance and a non-adjustable load reactance (Section 3.1), and then the power efficiency $\eta_{\Delta r}$ for the case of a non-adjustable load resistance and adjustable load reactance (Section 3.2). A general formulation of the power efficiency $\eta$ is then obtained from $\eta_{\Delta x}$ and $\eta_{\Delta r}$ using the load optimization factors.

\subsection{Non-adjustable load reactance}

We first assume that the load reactance has a deviation from its optimal value, and obtain both the value of resistance $r_{\text {subopt }}$ that maximizes efficiency, and the power efficiency $\eta_{\Delta x}$ obtained with $r=r_{\text {subopt }}$.

The optimal load resistance $r_{\text {subopt }}$ when the load reactance has a deviation $\Delta x\left(\Delta x=x-x_{\text {opt }}\right)$ can be obtained from Eq. (3) by forcing $\partial \eta / \partial r=0$, which results in:

$$
\begin{aligned}
r_{\text {subopt }} & =\frac{\sqrt{\left(r_{11} r_{22}-r_{\mathrm{m}}^{2}\right)\left(r_{11} r_{22}+x_{\mathrm{m}}^{2}\right)+\left(r_{11} \Delta x\right)^{2}}}{r_{11}} \\
& =\frac{|\boldsymbol{R}|}{r_{11}} \sqrt{1+(k Q)^{2}+\left[\frac{r_{11}}{|\boldsymbol{R}|}(\Delta x)\right]^{2}} .
\end{aligned}
$$




$$
\begin{aligned}
\eta & =\frac{(k Q)^{2}}{\left(1+\sqrt{1+(k Q)^{2}}\right)^{2}+\frac{r_{11}}{|\boldsymbol{R}|}\left(\sqrt{r}-\frac{r_{\text {subopt }}}{\sqrt{r}}\right)^{2}+\frac{2 r_{11}\left(r_{\text {subopt }}-r_{\mathrm{opt}}\right)}{|\boldsymbol{R}|}} \\
& =\frac{(k Q)^{2}}{\left(1+\sqrt{1+(k Q)^{2}}\right)^{2}+\sqrt{1+(k Q)^{2}}\left[\frac{1}{r_{\mathrm{opt}}}\left(\sqrt{r}-\frac{r_{\text {subopt }}}{\sqrt{r}}\right)^{2}+\frac{2\left(r_{\text {subopt }}-r_{\mathrm{opt}}\right)}{r_{\mathrm{opt}}}\right]} \\
& =\frac{(k Q)^{2}}{\left(1+\sqrt{1+(k Q)^{2}}\right)^{2}+\sqrt{1+(k Q)^{2}}\left(O_{\text {real }}^{2}+O_{\mathrm{imag}}^{2}\right)}
\end{aligned}
$$

Comparing the obtained $r_{\text {subopt }}$ with $r_{\text {opt }}$ in Eq. (4), we see that

$$
r_{\text {subopt }}^{2}=r_{\text {opt }}^{2}+(\Delta x)^{2} .
$$

Therefore, in an arbitrary two-terminal pair network, $r_{\text {subopt }}$ can also be calculated as

$$
r_{\text {subopt }}=\sqrt{r_{\mathrm{opt}}^{2}+(\Delta x)^{2}}
$$

Eqs. (11) and (12) tell us that $r_{\text {subopt }}$ is never smaller than $r_{\mathrm{opt}}$, and that these two values will be equal only when $x=$ $x_{\text {opt }}$.

The power efficiency $\eta_{\Delta x}$ with load impedance $z=$ $r_{\text {subopt }}+\mathrm{j}\left(x_{\text {opt }}+\Delta x\right)$ can be calculated as

$$
\eta_{\Delta x}=\frac{(k Q)^{2}}{\left(1+\sqrt{1+(k Q)^{2}}\right)^{2}+\frac{2 r_{11}\left(r_{\text {subopt }}-r_{\mathrm{opt}}\right)}{|\boldsymbol{R}|}}
$$

by substituting Eq. (12) into Eq. (3). Eqs. (8) and (13) show that the difference between $\eta_{\text {opt }}$ and $\eta_{\Delta x}$ lies only in the term $2 r_{11}\left(r_{\text {subopt }}-r_{\text {opt }}\right) /|\boldsymbol{R}|$ added to the denominator of $\eta_{\Delta \mathrm{x}}$.

\subsection{Non-adjustable load resistance}

Let us now derive the optimal load reactance $x_{\text {subopt }}$ and the power efficiency $\eta_{\Delta r}$ when the load resistance value $r$ deviates from its optimal value. Forcing $\partial \eta / \partial x=0$ in Eq. (3), $x_{\text {subopt }}$ is obtained as

$$
x_{\text {subopt }}=x_{\mathrm{opt}} .
$$

We therefore conclude that the optimal load reactance remains unaffected when the load resistance $r$ deviates from its optimal value.

The power efficiency $\eta_{\Delta r}$ with load impedance $z=r+$ j $x_{\text {subopt }}$ can be obtained by substituting Eq. (14) into Eq. (3):

$$
\begin{aligned}
\eta_{\Delta r} & =\frac{(k Q)^{2}}{2+(k Q)^{2}+\frac{r_{11}}{|\boldsymbol{R}|}\left(r+\frac{r_{\mathrm{opt}}^{2}}{r}\right)} \\
& =\frac{(k Q)^{2}}{\left(1+\sqrt{1+(k Q)^{2}}\right)^{2}+\frac{r_{11}}{|\boldsymbol{R}|}\left(\sqrt{r}-\frac{r_{\mathrm{opt}}}{\sqrt{r}}\right)^{2}} .
\end{aligned}
$$

The expression for $\eta_{\Delta r}$ is also very similar to that of $\eta_{\text {opt }}$, from which it can be obtained by adding the term $r_{11}\left(\sqrt{r}-r_{\text {opt }} / \sqrt{r}\right)^{2} /|\boldsymbol{R}|$ to the denominator. We also note that the terms added to the denominator of $\eta_{\text {opt }}$ to obtain the formulas for $\eta_{\Delta r}$ and $\eta_{\Delta x}$ have in common the fact of possessing a factor $r_{11} /|\boldsymbol{R}|$. The efficiency deteriorates as the load resistance value $r$ deviates from its optimal value $r_{\text {opt }}$; the same trend appears when the load reactance deviates from its optimal value.

\subsection{General formulation}

We now extend the above results and obtain an expression for $\eta$ in the general case of an arbitrary load impedance $z$.

By transforming Eq. (3) using a procedure similar to the one used to derive Eqs. (13) and (15), and using the fact that $\eta_{\Delta r}$ and $\eta_{\Delta x}$ have a common factor, the efficiency $\eta$ can be written as in Eq. (16).

Furthermore, we can delete $r_{11} /|\boldsymbol{R}|$ from Eq. (16) by using Eq. (4), and transform Eq. (16) into Eq. (17). When $z=$ $r_{\text {subopt }}+\mathrm{j} x$, Eq. (16) reduces to Eq. (13), as can be seen by using $r=r_{\text {subopt }}$ in Eq. (16). Hence, Eq. (16) is a general form of Eq. (13). Additionally, when $z=r+\mathrm{j} x_{\mathrm{opt}}$, $r_{\text {subopt }}=r_{\text {opt }}$ as per Eq. (12), and by replacing $r_{\text {subopt }}$ with $r_{\text {opt }}$, Eq. (16) becomes Eq. (15). This confirms that Eq. (16) is indeed a general form of both Eq. (13) and Eq. (15).

Let us now define the load optimization factors, to be used as quantitative indices showing how close the load resistance and load reactance are to their optimal values $r_{\text {subopt }}$ and $x_{\text {subopt }}=x_{\text {opt }}$, respectively. Specifically, two load optimization factors are defined: the load resistance optimization factor $O_{\text {real }}$, and load reactance optimization factor $O_{\text {imag }}$ :

$$
\begin{gathered}
O_{\text {real }}=\frac{\left|\sqrt{r}-\frac{r_{\text {subopt }}}{\sqrt{r}}\right|}{\sqrt{r_{\mathrm{opt}}}} \\
O_{\text {imag }}=\sqrt{\frac{2\left(r_{\text {subopt }}-r_{\mathrm{opt}}\right)}{r_{\mathrm{opt}}} .}
\end{gathered}
$$

Using the load optimization factors $O_{\text {real }}$ and $O_{\text {imag }}, \eta$ can be finally formulated as in Eq. (18).

As can be seen from Eq. (19), $O_{\text {real }}$ becomes smaller as $r$ approaches $r_{\text {subopt }}$, and equals zero only when $r=r_{\text {subopot }}$. When $O_{\text {real }}$ increases (as $r$ deviates from $r_{\text {subopt }}$ ), $\eta$ degrades. 
An important point is that $O_{\text {real }}$ can become zero if one can adjust $r$, even though $O_{\text {real }}$ is not independent of $x$. In other words, when $r$ is fully adjustable, $O_{\text {real }}$ can be brought to zero regardless of $\boldsymbol{Z}$ and the load reactance $x$. Therefore, $O_{\text {real }}$ is an appropriate optimization factor for the load resistance $r$.

The same is true for the load reactance optimization factor $O_{\text {imag }}$. It becomes smaller as $x$ approaches $x_{\text {subopt }}=x_{\text {opt }}$, and becomes zero only when $x=x_{\text {opt }}$, as can be seen from Eq. (20). In addition, $O_{\text {imag }}$ is independent of $r$, and for a given $\boldsymbol{Z}$ it is a function of only $x$ and can be brought to zero by adjusting $x$.

Leu us now consider the behavior of $O_{\text {real }}$ and $O_{\text {imag }}$ in the vicinity of $z=z_{\text {opt }}$. Assuming that $\Delta r=r-r_{\text {subopt }}$ and $\Delta x$ are much smaller than $r_{\mathrm{opt}}$ and, therefore, $\Delta r / r_{\mathrm{opt}}$ and $\Delta x / r_{\text {opt }}$ are much smaller than one, $O_{\text {real }}$ and $O_{\text {imag }}$ can be approximated as follows:

$$
\begin{gathered}
O_{\text {real }} \simeq\left|\frac{\Delta r}{r_{\text {opt }}}\right| \\
O_{\text {imag }} \simeq\left|\frac{\Delta x}{r_{\text {opt }}}\right| .
\end{gathered}
$$

Eqs. (21) and (22) tell us that $O_{\text {real }}$ and $O_{\text {imag }}$ represent the ratio of $\Delta r$ and $\Delta x$ to $r_{\text {opt }}$ in the vicinity of $z=z_{\text {opt }}$. Furthermore, $O_{\text {real }}^{2}+O_{\text {imag }}^{2}$ in Eq. (18) can then be approximated by

$$
O_{\text {real }}^{2}+O_{\text {imag }}^{2} \simeq\left|\frac{\Delta z}{r_{\text {opt }}}\right|^{2},
$$

where $\Delta z=\Delta r+\mathrm{j} \Delta x$. Hence, $O_{\text {real }}^{2}+O_{\text {imag }}^{2}$ is representing the squared value of the ratio of the load impedance deviation from $r_{\mathrm{opt}}$ in the vicinity of $z=z_{\mathrm{opt}}$. The above discussion supports the choice of $O_{\text {real }}$ and $O_{\text {imag }}$ as appropriate indices to show how optimized the load resistance and load reactance are.

$O_{\text {real }}$ and $O_{\text {imag }}$ make it possible to analyze how well the load resistance and load reactance optimized. For example, the comparison of the magnitude of $O_{\text {real }}$ and $O_{\text {imag }}$ tells us which the load resistance deviation or load reactance deviation dominantly deteriorates the power efficiency.

Finally we introduce the efficiency optimization ratio, defined as $\eta / \eta_{\text {opt }}$, and use it to calculate the reduction in power efficiency caused by a load impedance deviation. From Eq. (18),

$$
\begin{aligned}
\frac{\eta}{\eta_{\mathrm{opt}}} & =\left[1+\frac{\sqrt{1+(k Q)^{2}}}{\left(1+\sqrt{1+(k Q)^{2}}\right)^{2}}\left(O_{\text {real }}^{2}+O_{\text {imag }}^{2}\right)\right]^{-1} \\
& =\left[1+\frac{O_{\text {real }}^{2}+O_{\text {imag }}^{2}}{\left(\sqrt[4]{1+(k Q)^{2}}+1 / \sqrt[4]{1+(k Q)^{2}}\right)^{2}}\right]^{-1}
\end{aligned}
$$

is obtained. By introducing a complex factor $\chi$ defined as

$$
\chi=\frac{O_{\text {real }}+\mathrm{j} O_{\text {imag }}}{\sqrt[4]{1+(k Q)^{2}}+1 / \sqrt[4]{1+(k Q)^{2}}}
$$

the efficiency optimization ratio $\eta / \eta_{\mathrm{opt}}$ can be formulated in a quite simple form as

$$
\frac{\eta}{\eta_{\mathrm{opt}}}=\frac{1}{1+|\chi|^{2}} .
$$

Eq. (26) shows that the efficiency optimization ratio can be characterized by one single complex factor $(\chi)$. By calculating $\chi$, we can easily get to know how much the power efficiency deteriorated due to the load impedance deviation.

\section{Conclusion}

The analysis and optimization of the power efficiency of wireless power transfer systems using passive two-port network parameters has been hampered by the fact that these parameters change from moment to moment, leading to frequent mismatches between the determined optimal impedance and the actual load impedance and, consequently, to efficiency degradation. This paper modeled and analyzed the power efficiency of wireless power transfer systems and its associated efficiency optimization ratio using passive twoport network parameters, and defined two key load optimization factors - the load resistance optimization factor and the load reactance optimization factor-to indicate how much the load resistance and load reactance deviate from their optimal values. It was then shown that it is possible to express the power efficiency in terms of only the $k-Q$ product and those load optimization factors. Furthermore, it was shown that the efficiency optimization ratio can be characterized by one single complex factor. The formulation derived in this paper will be helpful to design engineers in estimating how much the power efficiency of these systems will deteriorate as a result of load impedance deviations.

\section{References}

[1] A. Kurs, et al.: "Wireless power transfer via strongly coupled magnetic resonances," Science 317 (2007) 83 (DOI: 10.1126/science. 1143254)

[2] A. Karalis, et al.: "Efficient wireless non-radiative mid-range energy transfer," Ann. Physics 323 (2008) 34 (DOI: 10.1016/j.aop.2007.04. 017)

[3] N. Shinohara: "Power without wires," IEEE Microw. Mag. 12 (2007) S64 (DOI: 10.1109/mmm.2011.942732)

[4] K. Takeno: "Wireless power transmission technology for mobile devices," IEICE Electron. Express 10 (2013) 20132010 (DOI: 10.1587/elex.10.20132010)

[5] M. Manoufali, et al.: "Wireless power link based on inductive coupling for brain implantable medical devices," IEEE Antennas Wireless Propag. Lett. 17 (2018) 160 (DOI: 10.1109/lawp.2017.2778698)

[6] C. Liu, et al.: "Circularly polarized implantable antenna for 915 MHz ISM-band far-field wireless power transmission," IEEE Antennas Wireless Propag. Lett. 17 (2018) 373 (DOI: 10.1109/lawp.2018. 2790418)

[7] A.P. Sample, et al.: "Analysis, experimental results, and range adaptation of magnetically coupled resonators for wireless power transfer," IEEE Trans. Ind. Electron. 58 (2010) 544 (DOI: 10.1109/tie.2010. 2046002)

[8] T. Imura and Y. Hori: "Maximizing air gap and efficiency of magnetic resonant coupling for wireless power transfer using equivalent circuit and Neumann formula," IEEE Trans. Ind. Electron. 58 (2011) 4746 (DOI: 10.1109/tie.2011.2112317)

[9] M. Kiani, et al.: "Design and optimization of a 3-coil inductive link for efficient wireless power transmission," IEEE Trans. Biomed. Circuits Syst. 5 (2011) 579 (DOI: 10.1109/tbcas.2011.2158431) 
[10] I. Awai: "Basic characteristics of "Magnetic resonance" wireless power transfer system excited by a $0 \mathrm{ohm}$ power source," IEICE Electron. Express 10 (2013) 20132008 (DOI: 10.1587/elex.10.20132008)

[11] Y. Narusue, et al:: "Impedance matching method for any-hop straight wireless power transmission using magnetic resonance," Proc. IEEE RWS (2013) 193 (DOI: 10.1109/rws.2013.6486685)

[12] S.Y.R. Hui, et al.: "A critical review of recent progress in mid-range wireless power transfer," IEEE Trans. Power Electron. 29 (2014) 4500 (DOI: 10.1109/tpel.2013.2249670)

[13] G. Monti, et al.: "Optimal design of a wireless power transfer link using parallel and series resonators," Wireless Power Transfer 3 (2016) 105 (DOI: 10.1017/wpt.2016.7)

[14] M. Zargham and P.G. Gulak: "Maximum achievable efficiency in near-field coupled power-transfer systems," IEEE Trans. Biomed. Circuits Syst. 6 (2012) 228 (DOI: 10.1109/tbcas.2011.2174794)

[15] T. Ohira: "Extended kQ product formulas for capacitive-and inductive-coupling wireless power transfer schemes," IEICE Electron. Express 11 (2014) 20140147 (DOI: 10.1587/elex.11.20140147)

[16] T. Ohira: "Maximum available efficiency formulation based on a black-box model of linear two-port power transfer systems," IEICE Electron. Express 11 (2014) 20140448 (DOI: 10.1587/elex.11. 20140448)

[17] H.D. Lang, et al.: "Convex optimization of wireless power transfer systems with multiple transmitters," IEEE Trans. Antennas Propag. 62 (2014) 4623 (DOI: 10.1109/tap.2014.2330584)

[18] Q.T. Duong and M. Okada: "Maximum efficiency formulation for inductive power transfer with multiple receivers," IEICE Electron. Express 13 (2016) 20160915 (DOI: 10.1587/elex.13.20160915)

[19] H. Ando, et al.: "Study on impedance matching and maximum wireless power transfer efficiency of circuits with resonant coupling based on simplified S-matrix," IEICE Electron. Express 16 (2019) 20190156 (DOI: 10.1587/elex.16.20190156)

[20] Y. Moriwaki, et al.: "Basic study on reduction of reflected power using DC/DC converters in wireless power transfer system via magnetic resonant coupling," Proc. IEEE INTELEC (2011) 1 (DOI: 10.1109/intlec.2011.6099737)

[21] M. Fu, et al.: "A cascaded boost-buck converter for high-efficiency wireless power transfer systems," IEEE Trans. Ind. Informat. 10 (2013) 1972 (DOI: 10.1109/tii.2013.2291682)

[22] W.X. Zhong and S.Y.R. Hui: "Maximum energy efficiency tracking for wireless power transfer systems," IEEE Trans. Power Electron. 30 (2014) 4025 (DOI: 10.1109/tpel.2014.2351496)

[23] $\mathrm{H}$. Li, et al.: "A maximum efficiency point tracking control scheme for wireless power transfer systems using magnetic resonant coupling," IEEE Trans. Power Electron. 30 (2015) 3998 (DOI: 10.1109/tpel.2014.2349534)

[24] Y. Narusue, et al:: "Maximizing the efficiency of wireless power transfer with a receiver-side switching voltage regulator," Wireless Power Transfer 4 (2017) 42 (DOI: 10.1017/wpt.2016.14)

[25] X. Dai, et al.: "Maximum efficiency tracking for wireless power transfer systems with dynamic coupling coefficient estimation," IEEE Trans. Power Electron. 33 (2017) 5005 (DOI: 10.1109/tpel.2017. 2729083)

[26] T.C. Beh, et al.: "Automated impedance matching system for robust wireless power transfer via magnetic resonance coupling," IEEE Trans. Ind. Electron. 60 (2013) 3689 (DOI: 10.1109/tie.2012. 2206337)

[27] Y. Lim, et al:: "An adaptive impedance-matching network based on a novel capacitor matrix for wireless power transfer," IEEE Trans. Power Electron. 29 (2014) 4403 (DOI: 10.1109/tpel.2013.2292596)

[28] J. Bito, et al.: "A novel heuristic passive and active matching circuit design method for wireless power transfer to moving objects," IEEE Trans. Microw. Theory Techn. 65 (2017) 1094 (DOI: 10.1109/tmtt. 2017.2672544)

[29] Z. Zhang, et al:: "Topology-reconfigurable capacitor matrix for encrypted dynamic wireless charging of electric vehicles," IEEE Trans. Veh. Technol. 67 (2018) 9284 (DOI: 10.1109/tvt.2018.2859779)

[30] D.M. Pozar: Microwave Engineering (Wiley, NJ, 2011) 4th ed. 192 (DOI: 10.1002/0471200549.ch8). 